\title{
The Conley index, cup-length and bifurcation
}

\author{
Zdzisław Dzedzej, Kazimierz Gȩba and Wojciech Uss
}

To Professor Richard Palais

\begin{abstract}
A module structure of the cohomology Conley index is used to define a relative cup-length. This invariant is applied then to prove a multiplicity theorem for periodic solutions to Hamiltonian systems.
\end{abstract}

Mathematics Subject Classification (2010). 37B30, 37J20, 37J45, 34C25, $58 \mathrm{E} 05$.

Keywords. Conley index, cup product, bifurcation, Hamiltonian system, periodic solution.

\section{Introduction}

In this paper, we consider a module structure of the Conley index of smooth flows in $\mathbb{R}^{n}$. If $\bar{\Omega}$ is an isolating neighbourhood and $\left(P_{1}, P_{2}\right)$ is a regular index pair in $\Omega$, then the cohomology $H^{*}\left(P_{1}, P_{2}\right)$ is a module over $H^{*}(\bar{\Omega})$. We define a notion of relative cup-length of $H^{*}\left(P_{1}, P_{2}\right)$ over $H^{*}(\bar{\Omega})$. This notion can be used to derive several results on nontrivial structure of invariant sets. As an example we prove a theorem on a minimal number of periodic solutions to Hamiltonian systems. A natural action of the group $S^{1}$ on the space of periodic functions is being used. Some other applications of this tool to bifurcation theory are presented in the PhD thesis of the last author [17].

It is worth mentioning that the concept is not completely new. One can find a cup-length applied to Conley theory in [2] and [4]. A variant of a relative version appeared in [16]. We believe that our approach should also be useful for other problems considered in the bifurcation theory.

The paper is organized as follows. Section 2 contains an abstract algebraic definition of the relative cup-length and simple properties. In Section 3 we recall basic concepts from Conley index theory (main source is [13]) and specify the abstract notion to it. In Section 4 we prove an abstract result on 
a number of critical points for gradient-like flows. Section 5 contains a reduction procedure for bifurcation problems. In the latter sections this procedure is applied to Hamiltonian systems.

\section{Relative cup-length}

Throughout this section we assume that $A \subset X \subset Y$ are compact metric spaces and we denote by $H^{*}$ the Alexander-Spanier cohomology with the coefficients in the fixed abelian group $G$. The cup product (see [15, Sec. 5.6])

$$
\smile: H^{k}(X) \times H^{l}(X, A) \rightarrow H^{k+l}(X, A)
$$

endows $H^{*}(X, A)$ with a structure of an $H^{*}(X)$-module. If $k: X \rightarrow Y$ denotes the inclusion map, then the formula

$$
\beta \cdot \alpha:=k^{*}(\beta) \smile \alpha
$$

defines on $H^{*}(X, A)$ a structure of $H^{*}(Y)$-module. The following remark is a simple consequence of the naturality property of the cup product (see $[6$, Prop. 3.10]).

Remark 2.1. If $B \subset A$ is compact, then

$$
H^{*}(X, A) \rightarrow H^{*}(X, B) \rightarrow H^{*}(A, B)
$$

is an exact sequence of $H^{*}(Y)$-modules, where the maps are induced by inclusions.

Definition 2.1. Let $\beta \in H^{p}(Y), p>0, \beta \neq 0$, and let $A \subset X \subset Y$ be CWcomplexes. The relative cup-length of $\beta$ with respect to $(X, A)$ is the number $\chi(\beta ; X, A) \in \mathbb{N}$ defined as follows:

- $\chi(\beta ; X, A)=0$ if $H^{*}(X, A)=0$;

- $\chi(\beta ; X, A)=1$ if $H^{*}(X, A) \neq 0$ and $\beta \cdot \alpha=0$ for every $\alpha \in H^{*}(X, A)$;

- $\chi(\beta ; X, A)=k \geq 2$ if there exists $\alpha_{0} \in H^{*}(X, A)$ such that $\beta^{k-1} \cdot \alpha_{0} \neq 0$ and $\beta^{k} \cdot \alpha=0$ for every $\alpha \in H^{*}(X, A)$.

Definition 2.2. The relative cup-length of the $H^{*}(Y)$-module $H^{*}(X, A)$ is the number given by

$$
\Upsilon(X, A ; Y):=\max \left\{\chi(\beta ; X, A) ; 0 \neq \beta \in H^{k}(Y), k>0\right\} .
$$

If $H^{k}(Y)=\{0\}$ for $k>0$ but $H^{*}(X, A)$ is nonzero, we set $\Upsilon(X, A ; Y)=1$, and if $H^{l}(X, A)$ are trivial for all $l \geq 0$, then $\Upsilon(X, A ; Y):=0$.

Lemma 2.2. If $B \subset A \subset X \subset Y$, then

$$
\Upsilon(X, B ; Y) \leq \Upsilon(X, A ; Y)+\Upsilon(A, B ; Y) .
$$

Proof. Let $k_{1}=\Upsilon(X, A ; Y), k_{2}=\Upsilon(A, B ; Y), 0 \neq \alpha \in H^{p}(X, B), p \geq 0$, $0 \neq \beta \in H^{q}(Y), q>0$. Consider the following inclusions:

$$
i:(X, B) \rightarrow(X, A), \quad j:(A, B) \rightarrow(X, B) .
$$


Since $k_{2}=\Upsilon(A, B ; Y), j^{*}\left(\beta^{k_{2}} \cdot \alpha\right)=0$. By Remark 2.1, there exists $\gamma \in$ $H^{*}(X, A)$ such that $\beta^{k_{2}} \cdot \alpha=i^{*}(\gamma)$. Therefore,

$$
\beta^{k_{1}+k_{2}} \cdot \alpha=i^{*}\left(\beta^{k_{1}} \cdot \gamma\right) \text {. }
$$

But $\beta^{k_{1}} \cdot \gamma=0$ by definition of $k_{1}$, and thus $\beta^{k_{1}+k_{2}} \cdot \alpha=0$. This means that

$$
\Upsilon(X, B ; Y) \leq k_{1}+k_{2},
$$

which ends the proof.

Lemma 2.3. If $A \subset X \subset Y_{1} \subset Y_{2}$, then

$$
\Upsilon\left(X, A ; Y_{2}\right) \leq \Upsilon\left(X, A ; Y_{1}\right) .
$$

Proof. Consider the following inclusions:

$$
s: X \hookrightarrow Y, \quad k: A \hookrightarrow X, \quad t: A \hookrightarrow Y .
$$

If $\beta \in H^{>0}\left(Y_{2}\right), \alpha \in H^{*}(X, A)$, then $\beta \alpha=t^{*}(\beta) \smile \alpha=k^{*}\left(s^{*}(\beta)\right) \smile \alpha$. Hence $\chi(X, A ; \beta)=\chi\left(X, A ; s^{*}(\beta)\right)$ for all $\beta \in H^{>0}\left(Y_{2}\right)$. Since $t=k \circ s$, the condition $t^{*}(\beta) \smile \alpha \neq 0$ implies $s^{*}(\beta) \smile \alpha \neq 0$, and our inequality follows.

Recall that the cross product is defined by the formula

$$
a \times b:=p_{1}^{*}(a) \smile p_{2}^{*}(b),
$$

where $p_{1}, p_{2}$ denote projections $(X, A) \times(Y, B)$ onto $(X, A)$ and $(Y, B)$. For algebraic properties of the maps

$$
\begin{aligned}
& \times: H^{k}(X ; R) \times H^{l}(Y ; R) \rightarrow H^{k+l}(X \times Y ; R), \\
& \times: H^{k}(X, A ; R) \times H^{l}(Y, B ; R) \rightarrow H^{k+l}(X \times Y, X \times B \cup A \times Y ; R)
\end{aligned}
$$

see, e.g., [6] or [1, pp. 240-242].

Let $\sigma:=$ generator $H^{1}(I, \partial I), I:=[-1,1]$.

The formula

$$
\mathfrak{S}(a):=a \times \sigma
$$

defines a mapping

$$
\mathfrak{S}: H^{k}(X, A) \rightarrow H^{k+1}((X, A) \times(I, \partial I))=H^{k+1}(X \times I, X \times \partial I \cup A \times I) .
$$

The following lemma holds (cf. [6, Thm. 3.21] for more general version).

Lemma 2.4. If $X \subset Y$, then $\mathfrak{S}$ is an isomorphism of $H^{*}(Y)$-modules. More exactly,

$$
\mathfrak{S}(b \cdot a)=p^{*}(b) \cdot \mathfrak{S}(a)
$$

where $p$ denotes the projection $Y \times I$ onto $Y$.

Proof. Let $b \in H^{*}(Y), a \in H^{*}(X, A)$. Consider the following projections:

$$
\begin{aligned}
& p_{1}:(X \times I, A \times I) \rightarrow(X, A), \\
& p_{2}:(X \times I, X \times \partial I) \rightarrow(I, \partial I), \\
& \bar{p}_{1}: X \times I \rightarrow X .
\end{aligned}
$$


The following diagram is commutative, where $i_{1}(x, t)=(i(x), t)$ :

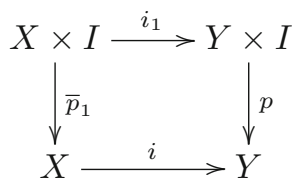

Using this diagram and the naturality and associativity properties of the cup product (see [1, p. 239]), we obtain

$$
\begin{aligned}
\mathfrak{S}(b \cdot a) & =(b \cdot a) \times \sigma=p_{1}^{*}\left(i^{*}(b) \smile a\right) \smile p_{2}^{*}(\sigma)=\bar{p}_{1}^{*}\left(i^{*}(b)\right) \smile p_{1}^{*}(a) \smile p_{2}^{*}(\sigma) \\
& =\bar{p}_{1}^{*}\left(i^{*}(b)\right) \smile \mathfrak{S}(a)=i_{1}^{*}\left(p^{*}(b)\right) \smile \mathfrak{S}(a)=p^{*}(b) \cdot \mathfrak{S}(a) .
\end{aligned}
$$

Theorem 2.5. The following formula holds:

$$
\Upsilon((X, A) \times(I, \partial I) ; Y)=\Upsilon(X, A ; Y)
$$

Proof. Let us notice that formally $X \times I \subset Y \times I$ and thus $H^{*}(X \times I, X \times$ $\partial I \cup A \times I)$ is an $H^{*}(Y \times I)$-module, but $p^{*}: H^{*}(Y) \rightarrow H^{*}(Y \times I)$ is an isomorphism which gives the naturally isomorphic $H^{*}(Y)$-module structure: $b \odot a:=p^{*}(b) \cdot a$ for $b \in H^{*}(Y)$ and $a \in H^{*}(X \times I, X \times \partial I \cup A \times I)$. Taking this into account, the desired equality follows directly from Lemma 2.4 .

\section{Conley index and the relative cup-length}

In this section, we recall the basic notions of the Conley index theory; the reader can refer to [9] and [13] for details. Let $X$ be a locally compact metric space. A continuous map $\eta: X \times \mathbb{R} \rightarrow X$ is a flow if it satisfies the conditions

$$
\begin{aligned}
\eta(x, 0) & =x, \\
\eta(x, t+s) & =\eta(\eta(x, t), s) .
\end{aligned}
$$

A set $S \subset X$ is an invariant set for the flow $\eta$ if

$$
\eta(S, \mathbb{R}):=\bigcup_{t \in \mathbb{R}} \eta(S, t)=S .
$$

For an arbitrary set $N \subset X$ one can define its invariant part

$$
\operatorname{Inv}(N, \eta):=\{x \in N \mid \eta(x, \mathbb{R}) \subset N\} .
$$

A compact set $N \subset X$ is an isolating neighbourhood if $\operatorname{Inv}(N, \eta) \subset \operatorname{int} N$. A set $S$ is called an isolated invariant set if there is an isolating neighbourhood $N$ such that $S=\operatorname{Inv}(N, \varphi)$. A flow $\eta: \mathbb{R} \times \mathbb{R}^{n} \rightarrow \mathbb{R}^{n}$ is generated by a smooth vector field $F: \mathbb{R}^{n} \rightarrow \mathbb{R}^{n}$ if $\eta(x, t)$ is the solution of the Cauchy problem $\dot{u}=-F(u), u(0)=x$ evaluated at time $t$. Such a flow is a gradient flow if $F=\nabla f$ for some smooth function $f: \mathbb{R}^{n} \rightarrow \mathbb{R}$.

Let $S$ be an isolated invariant set for the flow $\eta$. A compact pair $N_{0} \subset N_{1}$ of subsets of $X$ is called an index pair for $S$ if the following hold:

(a) $\overline{\operatorname{int}\left(N_{1} \backslash N_{0}\right)}$ is an isolating neighbourhood for $S$;

(b) $N_{0}$ is positively invariant relative to $N_{1}$; i.e., if $x \in N_{0}$ and $\eta(x,[0, t]) \subset$ $N_{1}$, then $\eta(x,[0, t]) \subset N_{0}$; 
(c) $N_{0}$ is an exit set for $N_{1}$; i.e., if $x \in N_{1}$ and $t_{1}>0$ such that $\eta\left(x, t_{1}\right) \notin N_{1}$, then there exists $t_{0} \in\left[0, t_{1}\right]$ for which $\eta\left(\left[0, t_{0}\right], x\right) \subset N_{1}$ and $\eta\left(x, t_{0}\right) \in$ $N_{0}$.

The following result implies the correctness of the definition of the homotopy Conley index (cf. [9, Thms. 2.2.1 and 2.2.2] or [13, Thms. 23.7 and $23.12])$.

Theorem 3.1. Let $S$ be an isolated invariant set for the flow $\eta$. Then there exists an index pair for $S$. Moreover, if $\left(N_{1}, N_{0}\right)$ and $\left(N_{1}^{\prime}, N_{0}^{\prime}\right)$ are index pairs for $S$, then the pointed topological spaces

$$
\left(N_{1} / N_{0},\left[N_{0}\right]\right) \quad \text { and } \quad\left(N_{1}^{\prime} / N_{0}^{\prime},\left[N_{0}^{\prime}\right]\right)
$$

are homotopically equivalent.

Definition 3.1. Let $S$ be an isolated invariant set for the flow $\eta$. The homotopy Conley index of $S$ is the homotopy type of the pointed space

$$
h(S)=h(S, \eta):=\left[N_{1} / N_{0},\left[N_{0}\right]\right]
$$

where $\left(N_{1}, N_{0}\right)$ is an index pair for $S$.

It is useful to consider the cohomology Conley index defined by

$$
C H^{*}(S):=H^{*}(N, L)=H^{*}(N / L),
$$

where $H^{*}$ denotes the Alexander-Spanier cohomology and $(N, L)$ is an index pair for $S$. The last equality means that we identify $H^{*}(N, L)$ and $H^{*}(N / L)$ via the isomorphism induced by the quotient map.

It is convenient to extend the index to an index of isolating neighbourhood: if $N$ is an isolating neighbourhood for $\eta$, then the homotopy (resp., cohomology) Conley index of $N$ is defined as

$$
\begin{gathered}
h(N)=h(N, \eta):=h(\operatorname{Inv}(N, \eta)), \\
\left(\operatorname{resp} ., C H^{*}(N)=C H^{*}(N, \eta):=C H^{*}(\operatorname{Inv}(N, \eta))\right) .
\end{gathered}
$$

Before giving the definition of the relative cup-length of Conley index, we need some useful lemmas. If $\left(N_{0}, N_{1}\right)$ is an index pair and $t \geq 0$, then, following [13], we set

$$
\begin{aligned}
N_{1}^{t}:= & \left\{x \in N_{1} ; \eta(x,[-t, 0]) \subset N_{1}\right\}, \\
N_{0}^{-t}:= & \left\{x \in N_{1} ; \text { there are } x^{\prime} \in N_{0} \text { and } t^{\prime} \in[0, t]\right. \\
& \text { with } \left.\eta\left(x^{\prime},\left[-t^{\prime}, 0\right]\right) \subset N_{1} \text { and } \eta\left(x^{\prime}, t\right)=x\right\} .
\end{aligned}
$$

For $t \geq 0$, define a map

$$
g: N_{1} / N_{0}^{-t} \rightarrow N_{1}^{t} /\left(N_{0} \cap N_{1}^{t}\right)
$$

by

$$
g([x]):= \begin{cases}{[\eta(x, t)]} & \text { if } \eta(x,[0, t]) \subset N_{1} \backslash N_{0} \\ * & \text { otherwise }\end{cases}
$$


It is known (see [13, Lem. 23.14]) that $g$ is a homeomorphism. Therefore, it induces an isomorphism

$$
g^{*}: H^{*}\left(N_{1}^{t}, N_{0} \cap N_{1}^{t}\right) \rightarrow H^{*}\left(N_{1}, N_{0}^{-t}\right) .
$$

Lemma 3.2. Assume that $N$ is an isolating neighbourhood for $\eta$ and $\left(N_{1}, N_{0}\right)$ is an index pair for $S \subset N$. If $N_{1} \subset N$, then the inclusion $i:\left(N_{1}, N_{0} \cap N_{1}^{t}\right) \rightarrow$ $\left(N_{1}, N_{0}^{-t}\right)$ induces an isomorphism

$$
i^{*}=\left(g^{*}\right)^{-1}: H^{*}\left(N_{1}, N_{0}^{-t}\right) \rightarrow H^{*}\left(N_{1}, N_{0} \cap N_{1}^{t}\right) .
$$

Proof. Consider the following diagram, where the vertical arrows denote the quotient maps.

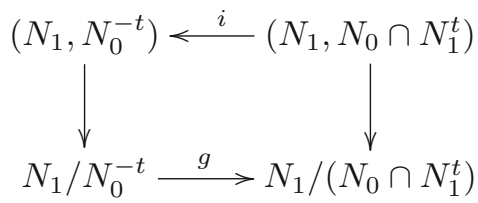

From the definition of $g$, it is obvious that the diagram is homotopy commutative and the conclusion follows.

Definition 3.2. Let $N$ be an isolating neighbourhood for the flow $\eta$. We define the relative cup-length of $\eta$ with respect to $N$ as

$$
\Upsilon(\eta, N):=\Upsilon\left(N_{1}, N_{0} ; N\right)
$$

where $\left(N_{1}, N_{0}\right)$ is an index pair for $S$.

The following lemma states that $\Upsilon(\eta, N)$ is well defined.

Lemma 3.3. Let $N$ be an isolating neighbourhood for $\eta$ and let $S \subset N$ be an isolated invariant set. If $\left(N_{1}, N_{0}\right)$ and $\left(\bar{N}_{1}, \bar{N}_{0}\right)$ are index pairs for $S$ such that $N_{1}, \bar{N}_{1} \subset N$, then

$$
\Upsilon\left(\bar{N}_{1}, \bar{N}_{0} ; N\right)=\Upsilon\left(N_{1}, N_{0} ; N\right) .
$$

Proof. As in the proof of [13, Lem. 23.17], we consider the following sequence of maps, where $j, \hat{i}, \hat{i}_{1}$ are defined by inclusion maps of pairs of spaces and $g, \hat{g}$ are as above. All of them are homotopy equivalences of pointed spaces, as it is proved in detail in [13]:

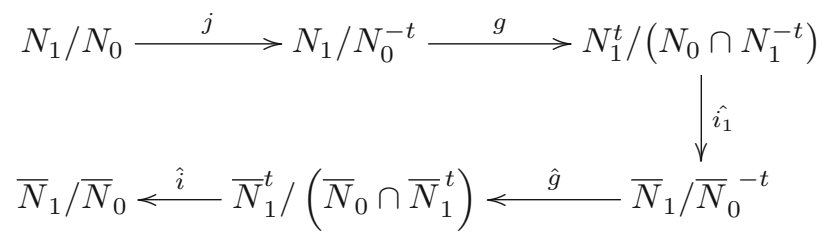


By Lemma 3.2 and definition of $j$, it follows that the following sequence of isomorphisms

$$
\begin{aligned}
& H^{*}\left(N_{1}, N_{0}\right) \longleftarrow H^{*}\left(N_{1}, N_{0}^{-t}\right) \longleftarrow \approx H^{*}\left(N_{1}^{t}, N_{0} \cap N_{1}^{-t}\right) \\
& \approx \uparrow \\
& H^{*}\left(\bar{N}_{1}, \bar{N}_{0}\right) \stackrel{\approx}{\longrightarrow} H^{*}\left(\bar{N}_{1}^{t}, \bar{N}_{0} \cap \bar{N}_{1}^{t}\right) \stackrel{\approx}{\longrightarrow} H^{*}\left(\bar{N}_{1}, \bar{N}_{0}^{-t}\right)
\end{aligned}
$$

are all induced by inclusions. Therefore, they all are isomorphisms of $H^{*}(N)$ modules and the conclusion follows.

One of the main properties of the Conley index is the continuation. The same holds true for the relative cup-length.

Lemma 3.4. Consider a continuous family of flows $\eta_{\lambda}: X \times \mathbb{R} \rightarrow X ; \lambda \in[0,1]$. Let $N \subset X$ be an isolating neighbourhood for all flows $\eta_{\lambda}$. Then

$$
\Upsilon\left(\eta_{0}, N\right)=\Upsilon\left(\eta_{1}, N\right)
$$

Proof. Similarly as in the proof of Lemma 3.3 we shall use parts of the proof of [13, Thm. 23.31]. Given $\mu \in[0,1]$, there exists a neighbourhood $W$ of $\mu$ in $[0,1]$ with the property that for all $\lambda \in W$, we can find pairs $\left(N_{1}, N_{0}\right) \subset\left(P_{1}^{\lambda}, P_{0}^{\lambda}\right) \subset\left(\overline{N_{1}}, \overline{N_{0}}\right)$ such that $\left(N_{1}, N_{0}\right),\left(\overline{N_{1}}, \overline{N_{0}}\right)$ are index pairs for $\eta_{\mu}$ in $N$, and $\left(P_{1}^{\lambda}, P_{0}^{\lambda}\right)$ is an index pair for $\eta_{\lambda}$ in $N$ (see [13, Lem. 23.28]). Then it is shown in the proof of [13, Thm. 23.31] that the inclusion $i:\left(N_{1}, N_{0}\right) \rightarrow\left(P_{1}^{\lambda}, P_{0}^{\lambda}\right)$ induces a homotopy equivalence of pointed spaces $N_{1} / N_{0}$ and $P_{1}^{\lambda} / P_{0}^{\lambda}$. The same argument applies to show that $i^{*}$ : $H^{*}\left(P_{1}^{\lambda}, P_{0}^{\lambda}\right) \approx H^{*}\left(N_{1}, N_{0}\right)$ is an isomorphism of $H^{*}(N)$-modules. Therefore, $\Upsilon\left(\eta_{\lambda}, N\right)=\Upsilon\left(\eta_{\mu}, N\right)$. Since $[0,1]$ is compact and connected, this completes the proof.

One easily sees that the continuation holds for more general parameter space $\Lambda$ as in [13].

\section{Gradient-like flows}

Throughout this section, as before, $\eta$ denotes a flow on a locally compact metric space $X$.

Let $N$ be an isolating neighbourhood for $\eta$ and let $\varphi: \operatorname{int} N \rightarrow \mathbb{R}$ be continuous. The flow $\eta$ is called gradient-like with respect to $\varphi$ if $\eta(x,[0, t]) \subset$ $\operatorname{int} N$ and $\eta(x, t) \neq x$ imply $\varphi(\eta(x, t))>\varphi(x)$. We define the critical level set of $\varphi$ with respect to $\eta$ as

$$
\operatorname{Crit}(\varphi, \eta):=\varphi(\{x \in U ; \eta(x, t)=x \text { for all } t \in \mathbb{R}\}) .
$$

In other words, $c \in \operatorname{Crit}(\varphi, \eta)$ if and only if there is $x \in N$ which is a rest point of the flow and $\varphi(x)=c$.

The aim of this section is to give a proof of the following theorem. 
Theorem 4.1. Assume that $X$ is locally contractible and $N$ is an isolating neighbourhood for $\eta$. If $\eta$ is gradient-like with respect to $\varphi: \operatorname{int} N \rightarrow \mathbb{R}$ and $\operatorname{Crit}(\varphi, \eta)$ is finite, then

$$
\# \operatorname{Crit}(\varphi, \eta) \geq \Upsilon(\eta, N)
$$

Before giving the proof of the theorem we shall recall some definitions and results concerning Morse decompositions.

Recall that the omega limit set of $x \in X$ is given by

$$
\omega(x):=\bigcap_{t>0} \operatorname{cl}(\eta(x,[t, \infty)))
$$

and the alpha limit set is

$$
\alpha(x):=\bigcap_{t<0} \operatorname{cl}(\eta(x,(-\infty, t])) .
$$

Assume that $S$ is an isolated invariant set for $\eta$. A Morse decomposition of $S$ is a finite collection, $\left\{M_{i}: 1 \leq i \leq n\right\}$, of disjoint compact invariant subsets of $S$ which can be ordered $\left(M_{1}, M_{2}, \ldots, M_{n}\right)$ in such a way that if $x \in S \backslash \bigcup\left\{M_{i}: 1 \leq i \leq n\right\}$, then there are indices $i<j$ such that $\omega(x) \subset M_{i}$ and $\alpha(x) \subset M_{j}$. Such an ordering will be called admissible. The elements $M_{i}$ of the Morse decomposition of $S$ will be called Morse sets of $S$. For an admissible ordering $\left(M_{1}, \ldots, M_{n}\right)$ of a Morse decomposition $S$, define subsets $M_{i j}, i<j$, by

$$
M_{i j}:=\left\{x \in S: \omega(x) \cup \alpha(x) \subset M_{i} \cup M_{i+1} \cup \cdots \cup M_{j}\right\} .
$$

The proof of the following existence theorem can be found in [13, Thm. 23.7] or in [12, Cor. 4.4].

Theorem 4.2. Let $S$ be an isolated invariant set for $\eta$ and $\left(M_{1}, M_{2}, \ldots, M_{n}\right)$ an admissible ordering of a Morse decomposition of $S$. Then there exists an increasing sequence of compact sets (a (Morse) filtration of $S$ ),

$$
N_{0} \subset N_{1} \subset \cdots \subset N_{n}
$$

such that for any $i<j$, the pair $\left(N_{j}, N_{i-1}\right)$ is an index pair for $M_{i j}$. In particular, $\left(N_{n}, N_{0}\right)$ is an index pair for $S$, and $\left(N_{j}, N_{j-1}\right)$ is an index pair for $M_{j}$.

Furthermore, given any isolating neighbourhood $N$ of $S$, and any neighbourhood $U$ of $S$, the sets $N_{j}$ can be chosen so that $\operatorname{cl}\left(N_{n} \backslash N_{0}\right) \subset U$ and each $N_{j}$ is positively invariant relative to $N$.

Proof of Theorem 4.1. Let

- $S:=\operatorname{Inv} N$;

- $\operatorname{Crit}(\varphi, \eta)=\left\{c_{1}<c_{2}<\cdots<c_{k}\right\}$;

- $M_{i}:=\operatorname{Crit}(\varphi, \eta) \cap \varphi^{-1}\left(c_{i}\right)$.

Choose

$$
N_{0} \subset N_{1} \subset \cdots \subset N_{n}
$$


satisfying the conditions of Theorem 4.2. Lemma 2.2 implies

$$
\Upsilon\left(N_{i}, N_{0} ; N\right) \leq \Upsilon\left(N_{i-1}, N_{0} ; N\right)+\Upsilon\left(N_{i}, N_{i-1} ; N\right)
$$

for $i=1,2, \ldots, k$. Since $M_{i}$ is finite and $X$ is locally contractible, we can find a neighbourhood $U \subset N$ of $M_{i}$ consisting of pairwise disjoint contractible sets. Then we find an index pair $\left(N_{i}^{\prime}, N_{i-1}^{\prime}\right)$ in $U$. Therefore, $H^{*}\left(N_{i}^{\prime}, N_{i-1}^{\prime}\right)$ has a trivial structure as an $H^{*}(N)$-module. Thus by Lemma 3.3, we obtain

$$
\Upsilon\left(N_{i}, N_{i-1} ; N\right) \leq 1
$$

Therefore,

$$
\Upsilon(\eta, N)=\Upsilon\left(N_{k}, N_{0} ; N\right) \leq k
$$

\section{Bifurcation}

Throughout this section we let $E_{1}, E_{0}$ be Banach spaces, $H$ a Hilbert space and we assume that $E_{1} \subset E_{0} \subset H$, where the embeddings are continuous.

We assume also that a compact Lie group $G$ acts orthogonally on $H$, and the action on $E_{1}, E_{0}$ is by isometries (i.e., the norms on $E_{1}, E_{0}$ are $G$-invariant).

Definition 5.1. Given an open $\Omega \subset E$ and a continuous $f: \Omega \rightarrow E_{0}$, we say that $f$ is a generalized gradient map if there is an open $\Omega_{0} \subset E_{0}$, with $\Omega \subset \Omega_{0}$, and a $C^{1}$-function $\varphi: \Omega_{0} \rightarrow \mathbb{R}$ such that

$$
D \varphi(x)(y)=\langle f(x), y\rangle \text { for all } x \in \Omega, y \in E_{0} .
$$

Here $\langle\cdot, \cdot\rangle$ denotes the scalar product in $H$. Similarly, in the case of an open $\Omega \subset E \times \mathbb{R}$ and a continuous $f: \Omega \rightarrow E_{0}$, we say that $f$ is a generalized gradient map if $f_{\lambda}: \Omega_{\lambda} \rightarrow E_{0}$ is a generalized gradient map. Here $\Omega_{\lambda}=\{x \in$ $E ;(x, \lambda) \in \Omega\}$.

If $X$ is a Banach space, we denote the open $\epsilon$-ball in $X$ by $B_{X}(\epsilon):=$ $\{x \in X ;\|x\|<\epsilon\}$ and $B_{X}\left(x_{0}, \epsilon\right):=\left\{x \in X ;\left\|x-x_{0}\right\|<\epsilon\right\}$.

If $V \subset E$ is a finite-dimensional linear subspace, then there is the orthogonal decomposition determined by $V$

$$
E_{1}=W_{1} \oplus V, \quad E_{0}=W_{0} \oplus V,
$$

where $W_{0}:=\left\{x \in E_{0} ;\langle x, y\rangle=0\right.$ for all $\left.y \in V\right\}, W_{1}:=E_{1} \cap W_{0}$.

Definition 5.2. Let $\left[\lambda_{1}, \lambda_{2}\right] \subset \mathbb{R}$. We say that a $C^{1}$-gradient equivariant map

$$
f: \Omega_{f} \rightarrow E_{0}
$$

where $\Omega_{f} \subset E \oplus \mathbb{R}$ is open $G$-invariant, $\{0\} \times\left[\lambda_{1}, \lambda_{2}\right] \subset \Omega_{f}$, defines a bifurcation problem on $\left[\lambda_{1}, \lambda_{2}\right]$ if

$$
f(0, \lambda)=0 \quad \text { for }(0, \lambda) \in \Omega_{f}
$$

and

$$
D_{x} f\left(0, \lambda_{i}\right): E \approx E_{0}, \quad i=1,2 .
$$

We shall also simply say that $f$ is a bifurcation problem. 
Definition 5.3. Let $f_{i}: \Omega_{i} \rightarrow E_{0}, i=1,2$, be two bifurcation problems on $\left[\lambda_{1}, \lambda_{2}\right]$. We say that $f_{1}$ and $f_{2}$ are equivalent if there exists an equivariant diffeomorphism

$$
\Psi: \Omega_{1} \rightarrow \Omega_{2}
$$

such that

$$
f_{2}=f_{1} \circ \Psi \text {. }
$$

Theorem 5.1. Let $f: \Omega_{f} \rightarrow E_{0}$ be a bifurcation problem on $\left[\lambda_{1}, \lambda_{2}\right]$. If there exist decompositions

$$
E_{1}=V \oplus W_{1}, \quad E_{0}=V \oplus W_{0}, \quad f(x, y, \lambda)=\left(f_{1}(x, y, \lambda), f_{2}(x, y, \lambda)\right),
$$

such that

$$
D f_{2}(0, \lambda)_{\mid W_{1}}: W_{1} \approx W_{0} \quad \text { for } \lambda \in\left[\lambda_{1}, \lambda_{2}\right]
$$

then there exist

(1) an open invariant $\Omega \subset \Omega_{f},\{0\} \times\left[\lambda_{1}, \lambda_{2}\right] \subset \Omega$;

(2) $g: \Omega_{g} \rightarrow E_{0}-a$ bifurcation problem on $\left[\lambda_{1}, \lambda_{2}\right]$;

such that

(a) $f_{\mid \Omega}$ is a bifurcation problem on $\left[\lambda_{1}, \lambda_{2}\right]$ equivalent to $g$;

(b) $g\left(V \cap \Omega_{g}\right) \subset V$ and $g^{-1}(0) \subset V$;

(c) if $D_{1} f_{2}(0,0, \lambda)=0$, then $D_{1} g(0,0, \lambda)=D_{1} f_{1}(0,0, \lambda)$.

The proof is based on the following two theorems.

Theorem 5.2 (Equivariant implicit function theorem). Let $V_{1}, V_{2}, W$ be Banach $G$-spaces, $\Omega \subset V_{1} \times V_{2}$ a G-invariant open set, $\left(x_{0}, 0\right) \in \Omega$ and $F: \Omega \rightarrow W$ be continuously differentiable $G$-map. Assume that $F\left(x_{0}, 0\right)=0$ and

$$
D_{2} F\left(x_{0}, 0\right): V_{2} \rightarrow W
$$

is a G-equivariant Banach space isomorphism. Then there exist $\epsilon_{1}, \epsilon_{2}>0$, $B_{V_{1}}\left(x_{0}, \epsilon_{1}\right) \times B_{V_{2}}\left(\epsilon_{2}\right) \subset \Omega$, and a continuously differentiable $G$-equivariant map $\psi: B_{V_{1}}\left(x_{0}, \epsilon_{1}\right) \rightarrow B_{V_{2}}\left(\epsilon_{2}\right)$ such that

$$
F(x, \psi(x))=0
$$

and

$$
D \psi(x)=-\left(D_{2} F(x, \psi(x))\right)^{-1} D_{1} F(x, \psi(x))
$$

for all $x \in B_{V_{1}}\left(x_{0}, \epsilon_{1}\right)$. Furthermore, for every $x \in B_{V_{1}}\left(x_{0}, \epsilon_{1}\right), \psi(x)$ is the only solution of $(3)$ in $B_{V_{2}}\left(\epsilon_{2}\right)$.

Proof. The theorem is an equivariant reformulation of [7, Thm. 10.1]. Since the mapping

$$
G(x, y):=y-L_{0}^{-1} F(x, y), \quad L_{0}:=D_{2} F\left(x_{0}, 0\right),
$$

defined in [7, p. 134], is in our case equivariant, the proof carries over directly. 
Theorem 5.3 (Parametrized equivariant implicit function theorem). Let $V_{1}$, $V_{2}, W$ be Banach $G$-spaces, $\Omega \subset V_{1} \times V_{2} \times \mathbb{R}$ a $G$-invariant open set, $(0,0, \lambda) \in$ $\Omega$ for $\lambda \in\left[\lambda_{1}, \lambda_{2}\right]$. Assume that $F: \Omega \rightarrow W$ is a continuously differentiable $G$-map, $F(0,0, \lambda)=0$ if $(0,0, \lambda) \in \Omega$ and

$$
D_{2} F(0,0, \lambda): V_{2} \rightarrow W
$$

is a $G$-equivariant Banach space isomorphism if $(0,0, \lambda) \in \Omega$. Then there exist $\epsilon_{1}, \epsilon_{2}>0, B_{V_{1}}\left(\epsilon_{1}\right) \times B_{V_{2}}\left(\epsilon_{2}\right) \times\left(\lambda_{1}-\epsilon_{1}, \lambda_{2}+\epsilon_{1}\right) \subset \Omega$, and a continuously differentiable $G$-equivariant map $\psi: B_{V_{1}}\left(\epsilon_{1}\right) \times\left(\lambda_{1}-\epsilon_{1}, \lambda_{2}+\epsilon_{1}\right) \rightarrow B_{V_{2}}\left(\epsilon_{2}\right)$ such that

$$
F(x, \psi(x, \lambda), \lambda)=0
$$

and

$$
D \psi(x, \lambda)=-\left(D_{2} F(x, \psi(x, \lambda))\right)^{-1} D_{1} F(x, \psi(x, \lambda))
$$

for all $x \in B_{V_{1}}\left(\epsilon_{1}\right) \times\left[\lambda_{1}, \lambda_{2}\right]$. Furthermore, for every $(x, \lambda) \in B_{V_{1}}\left(\epsilon_{1}\right) \times$ $\left[\lambda_{1}, \lambda_{2}\right], \psi(x, \lambda)$ is the only solution of $(5)$ in $B_{V_{2}}\left(\epsilon_{2}\right)$.

Proof. The theorem follows from Theorem 5.2. One should consider $V_{1} \oplus R$ instead of $V_{1}$ and then use the compactness of $\left[\lambda_{1}, \lambda_{2}\right]$.

Proof of Theorem 5.1. We apply Theorem 5.3 to the map $f_{2}$ and obtain a $G$-equivariant mapping

$$
\psi: B_{V}\left(\epsilon_{1}\right) \times\left(\lambda_{1}-\epsilon_{1}, \lambda_{2}+\epsilon_{1}\right) \rightarrow B_{W_{0}}\left(\epsilon_{2}\right) .
$$

Observe that for each $\lambda \in\left[\lambda_{1}, \lambda_{2}\right]$, the following holds true:

$$
\text { if } x \in B_{V}\left(\epsilon_{1}\right), y \in B_{W_{0}}\left(\epsilon_{2}\right) \quad \text { then } \quad f_{2}(x, y, \lambda)=0 \Longleftrightarrow y=\psi(x, \lambda) \text {. }
$$

Taking $\epsilon_{2}$ smaller if necessary, we define a $G$-equivariant diffeomorphism

$$
\Psi: B_{V}\left(\epsilon_{1}\right) \times\left(\lambda_{1}-\epsilon_{1}, \lambda_{2}+\epsilon_{1}\right) \rightarrow \Omega_{f}
$$

by the following formula:

$$
\Psi(x, y, \lambda):=(x, y+\psi(x, \lambda), \lambda) .
$$

The desired map $g$ is given by

$$
g:=f \circ \Psi
$$

Since $V$ is finite dimensional, for $\epsilon>0$ small enough, we have

$$
g^{-1}(0) \cap\left(B_{V}(\epsilon) \times B_{W_{1}}(\epsilon) \times\left[\lambda_{1}, \lambda_{2}\right]\right) \subset B_{V}(\epsilon) \times\left[\lambda_{1}, \lambda_{2}\right] .
$$

Considering $D g(0,0)$ in a block form, we obtain the last assertion. 


\section{Bifurcation in $\mathbb{R}^{n}$}

In this section to simplify the notation, we consider a finite-dimensional bifurcation problem on $I=[-1,1]$ defined by a map $f$. More precisely, we assume that $f: \mathbb{R}^{n} \times \mathbb{R} \rightarrow \mathbb{R}^{n}$ is a $C^{1}$-map, $f(0, \lambda)=0$ for $\lambda \in \mathbb{R}$ and $\operatorname{Df}(0, \pm 1): \mathbb{R}^{n} \approx \mathbb{R}^{n}$.

Let $A_{\lambda}:=D_{x} f(0, \lambda)$. Then $f(x, \lambda)=A_{\lambda}(x)+f_{0}(x, \lambda)$. For $\tau \in[0,1]$, we set

$$
f_{\tau}(x, \lambda):=A_{\lambda}(x)+\tau f_{0}(x, \lambda) .
$$

Assume further that there exist $\rho, C>0$ such that

$$
\left\langle f_{\tau}(x, 1), x\right\rangle \geq C|x|^{2} \quad \text { for }|x| \leq 2 \rho
$$

and

$$
\left\langle f_{\tau}(x,-1), x\right\rangle \leq-C|x|^{2} \text { for }|x| \leq 2 \rho .
$$

For $\alpha>0$ and $0<\epsilon<\rho$, define

$$
F_{\tau}: \mathbb{R}^{n+1} \rightarrow \mathbb{R}^{n+1}
$$

by $F_{\tau}(x, \lambda):=\left(f_{\tau}(x, \lambda), \alpha(|x|-\epsilon)\right)$. Let

$$
\Omega=\left\{x \in \mathbb{R}^{n} ;|x| \leq 2 \rho\right\} \times[-1,1]
$$

and $M:=\sup \left\{\left|f_{\tau}(x, \lambda)\right| ;(x, \lambda) \in \Omega, \tau \in[0,1]\right\}$.

Lemma 6.1. If

$$
\alpha \geq \frac{2 M}{\rho(\rho-\epsilon)},
$$

then there exists $\delta>0$ such that $\delta<\epsilon$ and for all $\tau \in[0,1]$, the set $N:=$ $\{(x, \lambda) \in \Omega ;|x| \geq \delta\}$ is an isolating neighbourhood for the flow generated by $F_{\tau}$.

Proof. First we prove that $\Omega$ is an isolating neighbourhood. We fix $\tau$ and let $\eta(x, \lambda, t)=\left(\eta_{1}(x, \lambda, t), \eta_{2}(x, \lambda, t)\right) \in \mathbb{R}^{n} \times \mathbb{R}$ denote the flow generated by $F_{\tau}$. It is enough to show that for all $(x, \lambda) \in \partial \bar{\Omega}$,

(a) there exists $T>0$ such that either $\eta(x, \lambda, T) \notin \bar{\Omega}$ or $\eta(x, \lambda,-T) \notin \bar{\Omega}$.

Let $K:=\{(x, \lambda) \in \bar{\Omega} ;|x|=2 \rho, \lambda \in[-1,1]\}$. If $(x, \lambda) \in \partial \Omega \backslash K$, then (a) follows immediately from the definition of $F_{\tau}$.

To complete the proof of our first claim we start from the following simple observations:

(b) if $\eta(x, \lambda, t) \in \bar{\Omega}$ for all $t \in[0, T]$, then

$$
\left|\eta_{1}(x, \lambda, t)-\eta_{1}(x, \lambda, 0)\right| \leq T M \quad \text { for } t \in[0, T] ;
$$

(c) if $\eta(x, \lambda, t) \in A:=\{(x, \lambda) \in \bar{\Omega} ;|x| \geq \rho\}$ for all $t \in[0, T]$, then

$$
\eta_{2}(x, \lambda, t)-\eta_{2}(x, \lambda, 0) \geq(\rho-\epsilon) \alpha t \quad \text { for } t \in[0, T] .
$$


Let $\left(x_{0}, \lambda_{0}\right) \in K$ and

$$
T_{1}:=\inf \left\{t \in(0, \infty) ; \eta\left(x_{0}, \lambda_{0}, t\right) \notin A\right\} .
$$

Since every point of $A$ leaves $A$ in a finite time, $T_{1}<\infty$. (One can call $T_{1}$ the exit time of $\left(x_{0}, \lambda_{0}\right)$ from $A$.) Let $\left(x_{1}, \lambda_{1}\right):=\eta\left(x_{0}, \lambda_{0}, T_{1}\right)$. If $\left(x_{1}, \lambda_{1}\right) \in \partial \Omega$, then (a) holds. Suppose that $\left(x_{1}, \lambda_{1}\right) \in \Omega$. Then $\left|x_{1}\right|=\rho, \lambda_{1} \in(-1,1)$ and (b) implies $\rho \leq M T_{1}$. Applying (c), one obtains

$$
\lambda_{1} \geq \lambda_{0}+\alpha(\rho-\epsilon) \frac{\rho}{M}>\lambda_{0}+2>1 .
$$

We have obtained a contradiction. Therefore, $\Omega$ is an isolating neighbourhood for all $\eta_{\tau}$ and thus the invariant part

$$
\operatorname{Inv}(\Omega, \eta)=\bigcup_{\tau \in[0,1]} \operatorname{Inv}\left(\Omega, \eta_{\tau}\right) \subset \operatorname{int}(\Omega)
$$

is compact. Moreover, one easily checks that it is disjoint with $\{0\} \times[-1,1]$. Thus there exists $\epsilon>\delta \geq 0$ such that $\operatorname{Inv}(\Omega) \in \operatorname{int}(N)$. This proves that $N$ is an isolating neighbourhood for all $\eta_{\tau}$.

Assume now that $V=\left(\mathbb{R}^{n}, \varphi\right)$ is an orthogonal representation of a compact Lie group $G$; i.e., $\varphi: G \rightarrow O(n)$ is a group homomorphism. Let $S(V):=\{x \in V ;|x|=1\}$. The use of $V$ instead of $\mathbb{R}^{n}$ is a bit of notation abuse - we try to emphasize that $S(V)$ is a $G$-space.

Lemma 6.2. Let $f: \Omega_{f} \rightarrow \mathbb{R}^{n}$ be a gradient equivariant bifurcation problem on $[-1,1]$ and $A_{\lambda}:=D_{x} f(0, \lambda), \lambda \in[-1,1]$. Assume that there is $C>0$ such that

$$
\left\langle A_{1}(x), x\right\rangle \geq C|x|^{2} \quad \text { for } x \in \mathbb{R}^{n}
$$

and

$$
\left\langle A_{-1}(x), x\right\rangle \leq-C|x|^{2} \quad \text { for } x \in \mathbb{R}^{n} .
$$

Then for sufficiently small $\epsilon$, the number of zero $G$-orbits of $f$ in $S\left(\mathbb{R}^{n}, \epsilon\right) \times$ $(-1,1)$ is not less than the cup-length of $S(V) / G$.

Proof. We keep the notation from the beginning of this section. From (9) and (10), it follows that there exists $\rho>0$ such that assumptions (7) and (9) are satisfied. Now for $\epsilon<\rho$, we find $\alpha$ and $\delta$ as in Lemma 6.1 and obtain an isolating neighbourhood $N=\{(x, \lambda) ; \delta \leq|x| \leq 2 \rho,-1 \leq \lambda \leq 1\}$ which is clearly an invariant set with respect to the action of $G$ (trivial on the parameter space). By Lemma 3.4, it is enough to calculate the equivariant Conley index (and the relative cup-length) for the flow generated by the map $g(x, \lambda):=\left(D_{x} f(0, \lambda)(x), \alpha(|x|-\epsilon)\right)=\left(A_{\lambda} x, \alpha(|x|-\epsilon)\right)$.

Now we can make another simplification. Consider a map $B: \mathbb{R}^{n} \times \mathbb{R} \rightarrow$ $\mathbb{R}^{n}$ given by $B(x, \lambda)=\lambda x$ and a family of flows generated by vector fields $F_{\tau}: \mathbb{R}^{n+1} \rightarrow \mathbb{R}^{n+1}, F_{\tau}(x, \lambda)=\left(\tau A_{\lambda} x+(1-\tau) B(x, \lambda), \alpha(|x|-\epsilon)\right)$ with $\tau \in[0,1]$. It is easy to verify that $N$ is an isolating neighbourhood for this 
family of flows. Thus we can do all the calculations for $\tau=0$. We can easily find an index pair: $N_{1}:=N$ and

$$
\begin{aligned}
N_{0}:= & \{(x, 1): 1 \geq|x| \geq \epsilon\} \cup\{(x, \lambda) ;|x|=2 \rho, \lambda \in[0,1]\} \\
& \cup\{(x, \lambda) ;|x|=\delta, \lambda \in[-1,0]\} \\
& \cup\{(x,-1) ; \delta \leq|x| \leq \epsilon\} .
\end{aligned}
$$

Since all the sets are $G$-invariant, their quotient sets constitute an index pair for the flow generated on the orbit space. $N$ is equivariantly homotopy equivalent to $S(V) \times[-1,1] \times[-1,1]$ and $N_{0} \approx S(V) \times L$, where $L:\{(t, s) \in$ $\partial([-1,1] \times[-1,1]) ; t s \geq 0\}$. Therefore, $\overline{N_{1}}:=N_{1} / G \approx S(V) / G \times[-1,1] \times$ $[-1,1], \overline{N_{0}}:=N_{0} / G \approx S(V) / G \times L$. Their quotient $\overline{N_{1}} / \overline{N_{0}} \approx S(V) / G \wedge S^{1}$. Thus, by Theorem 2.5, $\Upsilon\left(\overline{N_{1}}, \overline{N_{0}} ; \overline{N_{1}}\right)$ is equal to the cup-length of $S(V) / G$.

Now we can apply Theorem 4.1, since the gradient flow generated by $f$ gives rise to a gradient-like flow on the orbit space and the critical points of this flow are images of the zero $G$-orbits of $f$.

\section{Bifurcations of periodic solutions to Hamiltonian systems} By

$$
J: \mathbb{R}^{2 N}=\mathbb{R}^{N} \oplus \mathbb{R}^{N} \rightarrow \mathbb{R}^{N} \oplus \mathbb{R}^{N}=\mathbb{R}^{2 N}
$$

we denote a linear automorphism given by the matrix

$$
\left(\begin{array}{cc}
0 & I \\
-I & 0
\end{array}\right)
$$

Throughout this section we assume that $H: \mathbb{R}^{2 N} \rightarrow \mathbb{R}$ is a $C^{2}$-function (Hamiltonian) such that

(H1) $H(0)=0, \nabla H(0)=0$;

(H2) the Hessian $\nabla^{2} H(0)$ is nondegenerate.

The main object of our investigation is periodic solutions to the following equation:

$$
\dot{u}(t)=J \nabla H(u(t)) .
$$

We shall use the following Banach spaces:

(1) $\mathcal{E}_{0}:=C\left(S^{1}, \mathbb{R}^{2 N}\right)$. The elements of $\mathcal{E}_{0}$ are identified with continuous functions

$$
u: \mathbb{R} \rightarrow \mathbb{R}^{2 N}, \quad u(t+2 \pi)=u(t), \quad\|u\|:=\sup \{|u(t)| ; t \in \mathbb{R}\} .
$$

(2) $\mathcal{E}:=C^{1}\left(S^{1}, \mathbb{R}^{2 N}\right)$. As a linear space $\mathcal{E}$ is a subspace of $\mathcal{E}_{0}$. The norm in $\mathcal{E}$ is defined by a formula

$$
\|u\|_{1}:=\|u\|+\|\dot{u}\| .
$$

The above automorphism $J$ defines also automorphisms of our Banach spaces

$$
J: \mathcal{E} \rightarrow \mathcal{E}, \quad J: \mathcal{E}_{0} \rightarrow \mathcal{E}_{0}
$$


More precisely,

$$
J\left(\sum_{i=1}^{2 N} u_{i} \mathbf{e}_{i}\right):=\sum_{i=1}^{2 N} u_{i} J\left(\mathbf{e}_{i}\right),
$$

where $\left\{\mathbf{e}_{1}, \mathbf{e}_{2}, \ldots, \mathbf{e}_{2 N}\right\}$ is the standard basis of $\mathbb{R}^{2 N}$.

In the space $\mathcal{E}_{0}$ we have a continuous inner product

$$
\langle u, v\rangle:=\sum_{j=1}^{2 N} \int_{0}^{2 \pi} u_{j}(t) v_{j}(t) d t
$$

where

$$
u=\sum_{j=1}^{2 N} u_{j} \mathbf{e}_{j}, \quad v=\sum_{j=1}^{2 N} v_{j} \mathbf{e}_{j} .
$$

(In other words, we consider $\mathcal{E}_{0}$ as a subspace of $\mathcal{L}^{2}\left(S^{1}, \mathbb{R}^{2 N}\right)$.)

The formula

$$
\mathcal{L}(u):=J(\dot{u})
$$

defines a bounded linear operator

$$
\mathcal{L}: \mathcal{E} \rightarrow \mathcal{E}_{0}
$$

Denote by

$$
\mathcal{H}: \mathcal{E} \rightarrow \mathcal{E}_{0}
$$

a map (nonlinear in general) given by a formula

$$
(\mathcal{H}(u))(t):=\nabla H(u(t)) .
$$

Our further considerations are based on the following well-known remark.

Define a map

$$
f: \mathcal{E} \times(0, \infty) \rightarrow \mathcal{E}_{0}, \quad f(u, \lambda):=\mathcal{L}(u)+\lambda \mathcal{H}(u) .
$$

Remark 7.1. A function $u \in \mathcal{E}$ is a periodic solution to equation (11) of period $\frac{2 \pi}{\lambda}$ if and only if $f(u, \lambda)=0$. The map $f$ is (generalized) gradient in the sense introduced in Definition 5.1 with respect to the potential $\chi(u):=\int_{0}^{2 \pi} u(t) d t$.

A change of variables $t \mapsto \lambda t$ gives the first part of the remark. The second part is well known.

Let $A:=\nabla^{2} H(0)$. The map $J A$ is a Hamiltonian (i.e., $(J A)^{T} J+$ $J(J A)=0)$. Observe that in [8] the notion Hamiltonian matrix is used.

Now we describe briefly the spectral decomposition of $J A$. We try to follow the notation of [8, Sec. 3.3], where further details can be found.

The eigenvalues of $J A$ fall into three groups (because of $(\mathrm{H} 2)$ ):

(1) the pure imaginary $\pm i \omega_{1}, \ldots, \pm i \omega_{r}$;

(2) the real eigenvalues $\alpha_{1}, \ldots, \alpha_{s}$;

(3) the truly complex $\pm \beta_{1} \pm i \gamma_{1}, \ldots, \pm \beta_{t} \pm i \gamma_{t}$. 
This defines a direct sum decomposition

$$
\mathbb{R}^{2 N}=\mathbb{V} \oplus \mathbb{X} \oplus \mathbb{Y},
$$

where their complexifications are composed of generalized eigenspaces as follows:

$$
\begin{aligned}
& \mathbb{V}^{c}=\bigoplus_{j=1}^{r}\left(\eta^{\dagger}\left(i \omega_{j}\right) \oplus \eta^{\dagger}\left(-i \omega_{j}\right)\right), \\
& \mathbb{X}^{c}=\bigoplus_{j=1}^{s}\left(\eta^{\dagger}\left(\alpha_{j}\right) \oplus \eta^{\dagger}\left(-\alpha_{j}\right)\right), \\
& \mathbb{Y}^{c}=\bigoplus_{j=1}^{t}\left(\eta^{\dagger}\left(\beta_{j}+i \gamma_{j}\right) \oplus \eta^{\dagger}\left(\beta_{j}-i \gamma_{j}\right) \oplus \eta^{\dagger}\left(-\beta_{j}+i \gamma_{j}\right) \oplus \eta^{\dagger}\left(-\beta_{j}-i \gamma_{j}\right)\right) .
\end{aligned}
$$

We are especially interested in part (1):

$$
\begin{aligned}
\sigma_{0}(J A) & =\sigma(J A) \cap\{i \mathbb{R}\} \\
& =\left\{ \pm i \omega_{1}, \pm i \omega_{2}, \ldots, \pm i \omega_{r}\right\}, \quad 0<\omega_{1}<\omega_{2}<\cdots<\omega_{r} .
\end{aligned}
$$

Denote by $\mathbb{V}_{j}, \mathbb{U}_{j}$ the subspaces of $\mathbb{R}^{2 N}$ such that

$$
\mathbb{V}_{j}^{c}:=\eta^{\dagger}\left(i \omega_{j}\right) \oplus \eta^{\dagger}\left(-i \omega_{j}\right), \quad \mathbb{U}_{j}^{c}:=\eta\left(i \omega_{j}\right) \oplus \eta\left(-i \omega_{j}\right) .
$$

Obviously,

$$
\mathbb{V}=\bigoplus_{j=1}^{r} \mathbb{V}_{j}
$$

and each summand is $A$-invariant (and so are $\mathbb{X}, \mathbb{Y}$ and $\mathbb{U}_{j}$ ).

Denote by $A^{c}: \mathbb{C}^{2 N} \rightarrow \mathbb{C}^{2 N}$ the complexification of $A$ and let $\mathbb{U}_{j} \subset \mathbb{R}^{2 N}$, $j=1, \ldots, r$, denote the subspace such that

$$
\mathbb{U}_{j}^{c}=\operatorname{Ker}\left(A+i \omega_{j}\right) \oplus \operatorname{Ker}\left(A-i \omega_{j}\right) .
$$

Let $d_{j}:=\frac{1}{2} \operatorname{dim} \mathbb{U}_{j}$. Clearly $d_{j}$ is an integer. Let

$$
d=d(A):=d_{1}+d_{2}+\cdots+d_{r} .
$$

In order to make our setup precise, we introduce the following terminology. If $u: \mathbb{R} \rightarrow \mathbb{R}^{2 N}$ is a periodic $C^{1}$-solution to (11) and $\tau \in \mathbb{R}$, then we let $u_{\tau}$ denote the periodic solution to (11) defined by $u_{\tau}(t):=u(t+\tau), t \in \mathbb{R}$. We say that two periodic solutions $u, v$ to (11) are geometrically distinct if $u_{\tau} \neq v$ for all $\tau \in \mathbb{R}$.

Now we can formulate the main result of this section.

Theorem 7.2. If $H$ satisfies (H1) and $(\mathrm{H} 2)$, then there exists an $\epsilon_{0}>0$ such that $0<\epsilon<\epsilon_{0}$ implies the existence of at least $d$ geometrically distinct periodic solutions to (11) in $\left\{u \in C\left(\mathbb{R}, \mathbb{R}^{2 N}\right) ;\|u\|=\epsilon\right\}$. 


\section{Proof of Theorem 7.2}

Define the operators $\mathcal{A}, \mathcal{D}_{\lambda}: \mathcal{E} \rightarrow \mathcal{E}_{0}$ by

$$
\mathcal{A}(u)(t):=A(u(t))=\left(\nabla^{2} H(0)\right)(u(t)), \quad \mathcal{D}_{\lambda} u:=J \dot{u}+\lambda A(u) .
$$

Note that $\mathcal{D}_{\lambda}=\operatorname{Df}(0, \lambda)$. For any subspace $\mathbb{Z} \subset \mathbb{R}^{2 N}$, we denote

$$
\mathcal{E}(\mathbb{Z}):=C^{1}\left(S^{1}, \mathbb{Z}\right), \quad \mathcal{E}_{0}(\mathbb{Z}):=C^{0}\left(S^{1}, \mathbb{Z}\right) .
$$

We consider the above function spaces together with the $S^{1}$-action defined by

$$
(\gamma u)(t):=u(t-\theta) \text { for } \gamma:=e^{i \theta}
$$

Notation. Throughout this section we tacitly assume that the considered maps are $S^{1}$-equivariant and gradient (in the generalized sense, see Definition 5.1). The gradient structure should be clear from the context.

Define an equivalence relation in the set $\mathfrak{S}:=\left\{\omega_{1}, \omega_{2}, \ldots, \omega_{q}\right\}$ by

$$
\omega_{j} \sim \omega_{k} \Longleftrightarrow n \omega_{j}=m \omega_{k}, \quad n, m \in \mathbb{N} .
$$

This relation divides $\mathfrak{S}$ into pairwise disjoint classes

$$
\mathfrak{S}=\bigcup_{k=1}^{p} \mathfrak{S}_{k}
$$

For $k \in\{1,2, \ldots, q\}$, set $\mathcal{J}_{k}:=\left\{j \in\{1, \ldots, r\} ; \omega_{j} \in \mathfrak{S}_{k}\right\}, \mathfrak{D}_{k}:=\mathcal{D}_{\lambda_{k}}$,

$$
\mathbb{W}_{k}:=\bigoplus_{j \in \mathcal{J}_{k}} \mathbb{V}_{j}, \quad b_{k}:=\sum_{j \in \mathcal{J}_{k}} d_{j},
$$

$\mathcal{W}_{k}:=\mathcal{E}\left(\mathbb{W}_{k}\right) \cap \operatorname{Ker} \mathfrak{D}_{k}$.

For each $k$, let $\nu_{k}$ denote the greatest real number such that for every $\omega \in \mathfrak{S}_{k}$ there is $n \in \mathbb{N}$ such that $\omega=n \nu_{k}$ and let $\lambda_{k}:=\nu_{k}^{-1}$.

Suppose $\omega_{j} \in \mathfrak{S}_{k}$ and let $n_{j}:=\frac{\omega_{j}}{\nu_{k}} \in \mathbb{N}$.

If $z=x+i y \in \mathbb{C}^{2 N}, x, y \in \mathbb{R}^{2 N}$, is an eigenvector corresponding to the eigenvalue $i \omega_{j}$ of $(J A)^{c}$, then $(J A)^{c}(x)=-\omega_{j} y,(J A)^{c}(y)=\omega_{j} x$ and thus $x-i y$ is an eigenvector corresponding to the eigenvalue $-i \omega_{j}$. Therefore, vectors $x, y$ span a subspace of $\mathbb{R}^{2 N}$ which is invariant for $A$. Let $\mathbf{z}_{p}=\mathbf{x}_{p}+i \mathbf{y}_{p}$, $p:=1, \ldots, d_{j}$, be a basis of $\operatorname{Ker}\left((J A)^{c}+i \omega_{j} I\right)$. Then the vectors

$$
\mathbf{x}_{1}, \mathbf{y}_{1}, \mathbf{x}_{2}, \mathbf{y}_{2}, \ldots, \mathbf{x}_{d_{j}}, \mathbf{y}_{d_{j}}
$$

form a basis of $\mathbb{U}_{j}$. Let $\mathbf{c}_{j}(t):=\cos \left(n_{j} t\right), \mathbf{s}_{j}(t):=\sin \left(n_{j} t\right)$. Denote by $\mathcal{U}_{j}$ the $2 d_{j}$-dimensional subspace of $C^{1}\left(S^{1}, \mathbb{U}_{j}\right) \subset \mathcal{E}$ spanned by

$$
\mathbf{c}_{j} \mathbf{x}_{p}+\mathbf{s}_{j} \mathbf{y}_{p}, \quad \mathbf{s}_{j} \mathbf{x}_{p}-\mathbf{c}_{j} \mathbf{y}_{p}, \quad p=1, \ldots, d_{j} .
$$

Then

$$
\mathcal{U}_{j}=\mathcal{E}\left(\mathbb{V}_{j}\right) \cap \operatorname{Ker} \mathfrak{D}_{k} \quad \text { and } \quad \mathcal{W}_{k}=\bigoplus_{j \in \mathcal{J}_{k}} \mathcal{U}_{j}
$$


Remark 8.1. The assignments

$$
\mathbf{c}_{j} \mathbf{x}_{p}+\mathbf{s}_{j} \mathbf{y}_{p} \mapsto e_{p}, \quad \mathbf{s}_{j} \mathbf{x}_{p}-\mathbf{c}_{j} \mathbf{y}_{p} \mapsto i e_{p}, \quad p=1, \ldots, d_{j},
$$

where $\left\{e_{p}\right\}$ denotes the standard basis of $\mathbb{C}^{d_{j}}$, define an isomorphism of real linear spaces

$$
\mathcal{A}_{j}: \mathcal{U}_{j} \rightarrow \mathbb{C}^{d_{j}}
$$

Lemma 8.2. The cup-length of $S\left(\mathcal{W}_{k}\right)$ equals $b_{k}$.

Proof. Consider the complex linear space

$$
\mathbf{V}:=\bigoplus_{j \in \mathcal{J}_{k}} \mathbb{C}^{d_{j}}
$$

whose points we write as $z=\left(z_{1}, \ldots, z_{q}\right), z_{j} \in \mathbb{C}^{d_{j}}$. Let $\mathbf{Y}$ and $\mathbf{Z}$ denote, respectively, the representations of $S^{1}$ on $\mathbf{V}$ determined by

$$
\begin{aligned}
& \gamma\left(z_{1}, \ldots, z_{q}\right):=\left(\gamma^{d_{1}} z_{1}, \ldots, \gamma^{d_{q}} z_{q}\right), \\
& \gamma\left(z_{1}, \ldots, z_{q}\right):=\left(\gamma^{b_{k}} z_{1}, \ldots, \gamma^{b_{k}} z_{q}\right) .
\end{aligned}
$$

To avoid misunderstandings we denote by $\mathbf{X}$ the standard representation of $S^{1}$ on $\mathbf{V}$. Let $\alpha: S(\mathbf{X}) \rightarrow S(\mathbf{Y}), \beta: S(\mathbf{Y}) \rightarrow S(\mathbf{Z})$ denote the $S^{1}$-equivariant maps between unit spheres in corresponding representations defined by

$$
\begin{aligned}
\alpha\left(z_{1}, \ldots, z_{q}\right) & :=\left(z_{1}^{d_{1}}, \ldots, z_{q}^{d_{q}}\right), \\
\beta\left(z_{1}, \ldots, z_{q}\right) & :=\left(z_{1}^{b_{k}-d_{1}}, \ldots, z_{q}^{b_{k}-d_{q}}\right) .
\end{aligned}
$$

Obviously $S(\mathbf{X}) / S^{1}=C P^{d-1}$. A slight modification of the arguments given in $\left[6\right.$, Sec. 3.2] permits to prove that $S(\mathbf{Z})$ is diffeomorphic to $C P^{a-1}$ and $\beta \circ \alpha$ induces a monomorphism of cohomology rings

$$
(\beta \circ \alpha)^{*}: H^{*}\left(S(\mathbf{Z}) / S^{1}\right) \rightarrow H^{*}\left(S(\mathbf{X}) / S^{1}\right) .
$$

Therefore,

$$
\alpha^{*}: H^{*}\left(S(\mathbf{Y}) / S^{1}\right) \rightarrow H^{*}\left(S(\mathbf{X}) / S^{1}\right)
$$

is also a monomorphism. Thus the cup-length of $S(\mathbf{Y})$ equals $b_{k}$. Since

$$
\bigoplus_{j \in \mathcal{J}_{k}} \mathcal{A}_{j}: \mathcal{W}_{k} \rightarrow \mathbf{Y}
$$

is an isomorphism of real representations of $S^{1}$, the proof is completed.

Let

$$
\begin{aligned}
\mathcal{W}_{k}^{\perp, 0} & :=\left\{w \in \mathcal{E}_{0}\left(W_{k}\right) ;\langle w, v\rangle=0 \text { for } v \in \mathcal{W}_{k}\right\} \\
\mathcal{W}_{k}^{\perp} & :=\mathcal{E}\left(\mathbb{W}_{k}\right) \cap \mathcal{W}_{k}^{\perp, 0} .
\end{aligned}
$$

From (12) and (18), we have

$$
\left\langle u, \mathfrak{D}_{k}(v)\right\rangle=\left\langle\mathfrak{D}_{k}(u), v\right\rangle=0 \quad \text { for } u \in \mathcal{W}_{k}, v \in \mathcal{E}\left(\mathbb{W}_{k}\right) .
$$


Therefore, $\mathfrak{D}_{k}\left(\mathcal{W}_{k}^{\perp}\right) \subset \mathcal{W}_{k}^{\perp, 0}$. Since $\mathfrak{D}_{k}$, as an operator from $\mathcal{E}\left(\mathbb{W}_{k}\right)$ into $\mathcal{E}_{0}\left(\mathbb{W}_{k}\right)$, is Fredholm of index 0 , it maps isomorphically $\mathcal{W}_{k}^{\perp}$ onto $\mathcal{W}_{k}^{\perp, 0}$. Applying Theorem 5.1, we obtain an $\epsilon>0$ and a mapping

$$
g: B(\mathcal{E}, \epsilon) \times\left[\lambda_{k}-\delta, \lambda_{k}+\delta\right] \rightarrow \mathcal{E}_{0},
$$

where $B(\mathcal{E}, \epsilon):=\{u \in \mathcal{E} ;\|u\|<\epsilon\}$, such that

- $f$ and $g$ determine equivalent bifurcation problems on $\left[\lambda_{k}-\delta, \lambda_{k}+\delta\right]$;

- $g\left(B\left(\mathcal{W}_{k}, \epsilon\right) \times\left[\lambda_{k}-\delta, \lambda_{k}+\delta\right]\right) \subset \mathcal{W}_{k}$

- $D g(0, \lambda)=D f(0, \lambda)$ for $\lambda \in\left[\lambda_{k}-\delta, \lambda_{k}+\delta\right]$.

Setting $\varphi(w, \lambda):=g(w, \lambda), w \in \mathcal{W}_{k}$, we obtain

$$
\varphi: \mathcal{W}_{k} \times\left[\lambda_{k}-\delta, \lambda_{k}+\delta\right] \rightarrow \mathcal{W}_{k}
$$

which determines a finite-dimensional bifurcation problem on $\left[\lambda_{k}-\delta, \lambda_{k}+\delta\right]$ (one may call it a reduction of $f$ to $\mathcal{W}_{k}$ ). Applying Lemmas 8.2 and 6.2, we obtain the following conclusion.

Conclusion 8.3. For each $k \in\{1, \ldots, q\}$, there exist $\delta, \epsilon>0$ such that

(a) the mapping $\varphi$ defines a bifurcation problem on $\left[\lambda_{k}-\delta, \lambda_{k}+\delta\right]$;

(b) $f^{-1}(0) \cap\left(S(\mathcal{E}, \epsilon) \times\left[\lambda_{k}-\delta, \lambda_{k}+\delta\right]\right)$ contains at least $b_{k}$ different $S^{1}$-orbits.

Now, to complete the proof of Theorem 7.2 , it is enough to observe that, for sufficiently small $\delta$ and $\epsilon$, different $S^{1}$ orbits in

$$
f^{-1}(0) \cap\left(\bigcup_{k=1}^{q} S(\mathcal{E}, \epsilon) \times\left[\lambda_{k}-\delta, \lambda_{k}+\delta\right]\right)
$$

correspond to geometrically distinct solutions to (11).

\section{Acknowledgment}

This research was supported by the Polish Ministry of Higher Education grant number N N 201394037.

\section{References}

[1] M. Aguilar, S. Gitler and C. Prieto, Algebraic Topology from a Homotopical Viewpoint. Springer-Verlag, New York, 2002.

[2] T. Bartsch, Topological methods for variational problems with symmetries. Lecture Notes in Math. 1560, Springer-Verlag, Berlin, 1993.

[3] C. Conley, Isolated Invariant Sets and the Morse Index. CBMS Regional Conference Series in Mathematics 38, Amer. Math. Soc., Providence, RI, 1978.

[4] A. Floer, A refinement of the Conley index and an application to the stability of hyperbolic invariant sets. Ergodic Theory Dynam. Systems 7 (1987), 93103.

[5] K. Gȩba, M. Izydorek and A. Pruszko, The Conley index in Hilbert spaces and its applications. Studia Math. 134 (1999), 217-233.

[6] A. Hatcher, Algebraic Topology. Cambridge University Press, Cambridge, 2002. 
[7] J. Jost, Postmodern Analysis. 3rd ed., Springer-Verlag, Berlin, 2005.

[8] K. Meyer, G. Hall and D. Offin, Introduction to Hamiltonian Dynamical Systems and the N-Body Problem. 2nd ed., Appl. Math. Sci. 90, SpringerVerlag, New York, 2009

[9] K. Mischaikow, Conley index theory. In: Dynamical Systems (Montecatini Terme, 1994), Lecture Notes in Math. 1609, Springer-Verlag, Berlin, 1995, 119-207.

[10] M. Mrozek, J. Reineck and R. Srzednicki, The Conley index over a base. Trans. Amer. Math. Soc. 352 (2000), 4171-4194.

[11] J. Robbin and D. Salamon, Dynamical systems, shape theory and the Conley index. Ergodic Theory Dynam. Systems 8 (1988), 375-393.

[12] D. Salamon, Connected simple systems and the Conley index of isolated invariant sets. Trans. Amer. Math. Soc. 291 (1985), 1-41.

[13] J. Smoller, Shock Waves and Reaction-Diffusion Equations. 2nd ed., Springer-Verlag, New York, 1994.

[14] J. Smoller and A. G. Wasserman, Bifurcation and symmetry-breaking. Invent. Math. 100 (1990), 63-95.

[15] E. H. Spanier, Algebraic Topology. Corrected reprint of the 1966 original, Springer-Verlag, New York, 1982.

[16] R. Srzednicki, Ważewski method and Conley index. In: Handbook of Differential Equations, Elsevier/North-Holland, Amsterdam, 2004, 591-684.

[17] W. Uss, Applications of Conley index in bifurcation theory. PhD thesis, Gdańsk University, Poland, 2011 (in Polish).

Zdzisław Dzedzej

Gdańsk University of Technology

Faculty of Technical Physics and Applied Mathematics

ul. Narutowicza 11/12, 80-233 Gdańsk, Poland

e-mail: zdzedzej@mif.pg.gda.pl

Kazimierz Gȩba

Gdańsk University of Technology

Faculty of Technical Physics and Applied Mathematics

ul. Narutowicza 11/12, 80-233 Gdańsk, Poland

e-mail: geba@mif.pg.gda.pl

Wojciech Uss

Gdańsk University of Technology

Faculty of Technical Physics and Applied Mathematics

ul. Narutowicza 11/12, 80-233 Gdańsk, Poland

e-mail: wuss@mif.pg.gda.pl

Open Access This article is distributed under the terms of the Creative Commons Attribution Noncommercial License which permits any noncommercial use, distribution, and reproduction in any medium, provided the original author(s) and source are credited. 